\title{
Is there a perfect protocol for patients with low ovarian reserve: a retrospective study comparing antagonist or agonist protocol in patients with low ovarian reserve
}

\author{
Seema Rai ${ }^{1 *}$, Yasaswi Khandavalli ${ }^{1}$, Rama S. Lodha ${ }^{2}$ \\ ${ }^{1}$ Department of Obstetrics and Gynecology, MH Bhopal, Bairagarh, Bhopal, Madhya Pradesh, India \\ ${ }^{2}$ Department of Community Medicine, Gandhi Medical College, Bhopal, Madhya Pradesh, India
}

Received: 20 June 2020

Accepted: 26 June 2020

*Correspondence:

Dr. Seema Rai,

E-mail: seema73rai@hotmail.com

Copyright: ( $)$ the author(s), publisher and licensee Medip Academy. This is an open-access article distributed under the terms of the Creative Commons Attribution Non-Commercial License, which permits unrestricted non-commercial use, distribution, and reproduction in any medium, provided the original work is properly cited.

\section{ABSTRACT}

Background: The high prevalence of infertility has made it a major healthcare problem in the present era. A majority of patients presenting with infertility have poor ovarian reserve (POR). Patients with POR are challenging to treat due to reduced treatment success and high cycle cancellation rate as there is no uniform definition and treatment protocol for these patients. The present retrospective study was performed to compare the pregnancy outcome between a long agonist protocol and flexible antagonist protocol in patients with POR. Patients with AMH $\leq 1.5 \mathrm{ng} / \mathrm{mL}$ and AFC $\leq 4$ was included in the study. Controlled ovarian hyperstimulation is the basis of any in vitro fertilisation (IVF) procedure. There is no universally accepted ideal stimulation protocol for patients with POR, and it remains a challenge.

Methods: This was a retrospective study covering the period from May 2019 to March 2020. Ninety-nine patients with low ovarian reserve (AMH $\leq 1.5 \mathrm{ng} / \mathrm{mL}$ and $\mathrm{AFC} \leq 4)$ were included in the study. The patients underwent $\mathrm{GnRH}$ agonist/GnRH antagonist stimulation protocol using recombinant FSH. Demographic characteristics like age, BMI, duration of infertility was comparable. Total days of stimulation, total Gonadotropin dose used and clinical pregnancy rate in both the protocols was analyzed. Difference between the two groups was considered statistically significant at p-value $<0.05$.

Results: Fifty-three patients underwent antagonist stimulation protocol and forty-six long agonist protocol. The clinical pregnancy rate was 37.7\% (20/53) and 32.6\% (15/46) in antagonist and agonist protocol respectively (pvalue $=0.5983$ ). Pregnancy rate was higher in the antagonist group but the difference was not statistically significant.

Conclusions: Antagonist protocol could marginally increase pregnancy rate in patients with low ovarian reserve. However, patients with poor ovarian reserve require a tailor-made protocol.

Keywords: Controlled ovarian hyperstimulation, GnRH-agonist, GnRH-antagonist, In vitro fertilization, Poor ovarian reserve

\section{INTRODUCTION}

Every patient expects and desires to take a healthy baby home on completion of an in vitro fertilisation (IVF) cycle. However, this is not always possible because IVF results, even in the best settings will also not guarantee this outcome. The outcome of any IVF cycle depends not only on maternal age but also heavily relies on the number and quality of retrieved oocytes. The number of oocytes retrieved plays a critical role in the IVF outcome. ${ }^{1-4}$

The ovarian reserve represents a woman's reproductive potential and a predictor of the number and quality of 
oocytes retrieved during IVF. ${ }^{5-7}$ A woman is born with a fixed number of primordial follicles in the ovary that gradually deplete with age. The rate of depletion can vary among women. Women with poor ovarian reserve (POR) have fewer oocytes and a poor response to controlled ovarian hyperstimulation $(\mathrm{COH})$ compared with other women of the same age group.

As per the Bologna criteria, at least two of the following three characteristics must be present to make a diagnosis of POR: (1) advanced maternal age ( $\geq 40$ years) or any other risk factors for POR, (2) a previous POR $(\leq 3$ oocytes) with a conventional stimulation protocol, or (3) an abnormal ovarian reserve test (antral follicle count (AFC) $<5-7$ follicles, or anti-mullerian hormone (AMH) $<0.5-1.1 \mathrm{ng} / \mathrm{mL}$ ) However, this criterion is not universally accepted. There is no cut-off for the adequate number of retrieved oocytes, but generally a retrieval of approximately 10-15 oocytes per cycle is considered adequate. $^{1}$ An adequate number of oocytes provides a sufficient number of embryos for transfer or cryopreservation. Patients with POR form a majority (25\%-35\%) of infertile patients. ${ }^{8-11}$ Patients with POR are challenging to treat. ${ }^{12-14}$ The aetiology and treatment of POR is very perplexing. No consensus on the best treatment modality for patients with POR has been reached despite great developments in the field of reproductive medicine. Retrieval of less than four oocytes is considered a poor or sub-optimal response to a stimulation. ${ }^{15,16}$

Over the past few years, many tests have been developed to predict the ovarian reserve and ovarian responsiveness. Variables such as age, body mass index (BMI), basal estradiol, inhibin $\mathrm{B}$, and follicle stimulating hormone (FSH) are indirect and less reliable markers of ovarian reserve. ${ }^{17}$ The most predictive and reproducible results are those of antral follicle count (AFC) and anti-mullerian hormone $(\mathrm{AMH})$, although the superiority of each over the other is unknown. The outcome of the IVF cycle in poor responders can be dismal due to fewer retrieved oocytes. There are multiple $\mathrm{COH}$ protocols used in IVF for poor, normal, and hyper-responders. ${ }^{18}$ Different stimulation protocols and adjuncts have been attempted to increase the yield of retrieved oocytes in an IVF cycle. ${ }^{19,20}$ However, the best protocol and adjunct for a positive result is debatable..$^{21-23}$ The $\mathrm{COH}$ protocol is basically planned depending on the patient's demographic characteristics, ovarian reserve, and response in previous cycles (if available). ${ }^{24,25}$ The most commonly used protocols are the long protocol with gonadotropin-releasing hormone (GnRH) agonist and $\mathrm{GnRH}$ antagonist protocol. ${ }^{26}$

After the initial flare-up, the GnRH agonist causes desensitisation of the pituitary gland. The pituitary gland is largely exhausted of luteinising hormone ( $\mathrm{LH})$. There is minimal residual $\mathrm{LH}$ in circulation by the time $\mathrm{COH}$ with gonadotropins is initiated, and LH-induced adverse effect is largely negated.
Introduction of the GnRH antagonist was a major breakthrough in the field of assisted reproduction. The GnRH antagonist causes immediate pituitary suppression without the initial flare-up or oestrogen deficiency symptoms and is believed to result in shorter cycles, smaller dose of gonadotropins, and a reduced risk of ovarian hyperstimulation syndrome with agonist trigger. $^{23,27}$ Researchers showed great interest in the GnRH antagonist in poor responders due to simpler stimulation protocols and lower gonadotropin requirements, leading to reduced cost and shorter downtime between two cycles. The antagonist protocol also allows the assessment of ovarian reserve just prior to starting the stimulation. The limitation of this protocol is that it is started late in the cycle and might not be to suppress LH before it adversely affects follicle and egg development.

In this study, we compared the long GnRH agonist and GnRH antagonist protocols for patients with POR to find which protocol gave a better result (Figure 1).

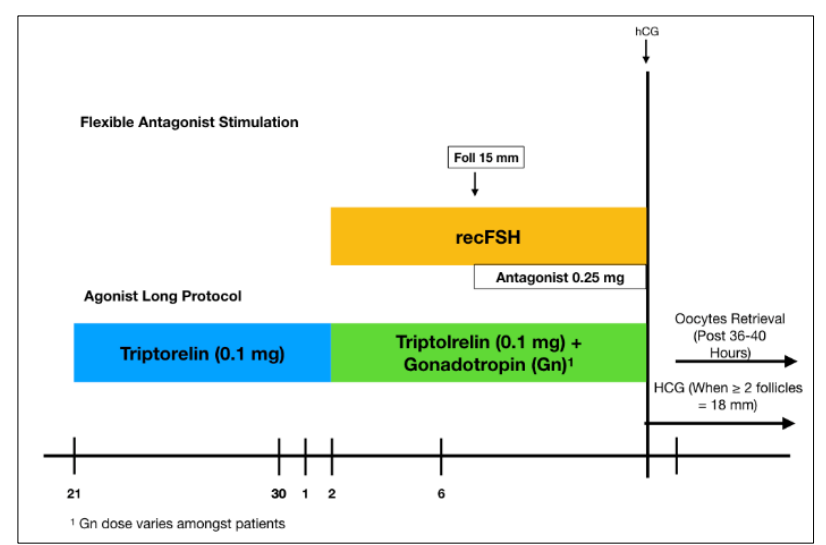

Figure 1: Flexible antagonist and agonist long controlled ovarian stimulation protocol.

\section{METHODS}

The present retrospective study was carried out in patients with POR who underwent IVF at a single centre from May 2019 to March 2020. A total of 356 patients underwent IVF out of which 99 had low ovarian reserve $(\mathrm{AMH} \leq 1.5 \mathrm{ng} / \mathrm{mL}$ and $\mathrm{AFC} \leq 4)$ and were included in the study. The GnRH antagonist protocol was carried out in 53 cycles and the GnRH agonist long protocol in 46 cycles. Patients with AMH less than $1.5 \mathrm{ng} / \mathrm{mL}$ and AFC less than 4 were considered as patients with POR and were included in the study.

\section{GnRH antagonist protocol}

On day 2-3 of the menstrual cycle, a baseline scan was performed to rule out ovarian cyst and assess the endometrium. Recombinant FSH (rFSH) (Gonal F, Merck Serono) at a dose of 225 IU was started daily. A transvaginal scan was carried out on day 5 to assess the 
follicular growth and endometrial thickness and repeated daily till the initiation of the GnRH antagonist. The GnRH antagonist, injection cetrorelix acetate (CETROLIX, INTAS Pharmaceuticals) $0.25 \mathrm{mg} /$ day, was added when the leading follicle was more than $1.4 \mathrm{~cm}$. The alternate day scan continued till the trigger was administered with 10,000 IU of human chorionic gonadotropin (FERTIGYN, Sun Pharma) (HCG) when the leading follicle was more than $1.8 \mathrm{~cm}$.

\section{GnRH agonist long protocol}

On day 2 of the cycle, a baseline transvaginal ultrasound examination was carried out to measure endometrial thickness and AFC. On day 5 of the menstrual cycle, patients were started on oral contraceptive pills for 21 days. On day 21 of the menstrual cycle, the GnRH agonist, triptorelin (Decapeptyl, Ferring) $(0.1 \mathrm{mg} / \mathrm{ml}$, was initiated. The patient was reviewed on day 2 of the menstrual cycle to ascertain pituitary downregulation. If the patient satisfied the criteria of downregulation (endometrial thickness $\leq 4 \mathrm{~mm}$, ovarian quiescence all follicle $\leq 10 \mathrm{~mm}$ ), $225 \mathrm{IU}$ of $\mathrm{rFSH}$ was started daily, and the agonist was continued. Alternate day transvaginal scans were continued from the 5th day of $\mathrm{COH}$ to monitor the follicular growth and endometrium till trigger. A trigger was given with 10,000 IU of HCG when the leading follicle was more than $1.8 \mathrm{~cm}$.

\section{Oocyte retrieval and $I V F$}

Oocyte retrieval was performed trans-vaginally under ultrasound guidance after $36-40 \mathrm{~h}$ of trigger. The retrieved oocytes were washed in a G-MOPS solution
(Vitrilife Sweden) and incubated for 2-3 hours in a GIVF solution (Vitrilife Sweden) in a humidified incubator at $6 \% \mathrm{CO}_{2}$ at $37^{\circ} \mathrm{C}$ followed by an intracytoplasmic sperm injection (ICSI).

\section{Embryo transfer}

Embryo transfer was done on day 3 at the 8-cell stage. A maximum of two grade 1 embryos were transferred, and the surplus were frozen. Grade 1 embryo was defined as possessing 6-8 blastomeres of equal size. Luteal phase support in the form of micronized progesterone injection and tablet dydrogesterone were started from the day of oocyte pick-up as per guidelines.

\section{Statistical analysis}

SPSS was used for statistical analysis. Data on age, basal FSH concentration, basal LH concentration, AMH, AFC, expected and retrieved number of oocytes, and dose of gonadotropins used were noted.

\section{RESULTS}

The age of patients included in the study varied between 22-44 years with an average age of $29.7 \pm 1.46$ years in the agonist protocol and $30.28 \pm 1.53$ years in the antagonist protocol. The duration of infertility in both groups were comparable $(6.56 \pm 4.3$ years in the agonist and $6.7 \pm 3.0$ years in the antagonist group). The dose of gonadotropin used and the number of stimulation days were comparable in both groups. Comparison of the demographic features of the two groups is summarised in Table 1.

Table 1: Demographic characteristics.

\begin{tabular}{|llll|}
\hline & AP & LP & Statistical difference \\
\hline Age (in years) & $29.7 \pm 1.46$ & $30.28 \pm 1.53$ & NS \\
\hline BMI $\left(\mathrm{kg} / \mathrm{m}^{2}\right)$ & $24.27 \pm 5.1$ & $23.7 \pm 4.2$ & NS \\
\hline Duration of infertility (years) & $6.7 \pm 3.0$ & $6.56 \pm 4.3$ & NS \\
\hline AFC & $3.18 \pm 0.44$ & $2.78 \pm 0.40$ & NS \\
\hline AMH $(\mathrm{ng} / \mathrm{mL})$ & $0.95 \pm 0.012$ & $0.87 \pm 0.15$ & NS \\
\hline Baseline FSH $(\mathrm{mIU} / \mathrm{mL})$ & $6.75 \pm 0.98$ & $7.43 \pm 0.94$ & NS \\
\hline Baseline $\mathrm{LH}(\mathrm{mIU} / \mathrm{mL})$ & $5.76 \pm 0.91$ & $5.76 \pm 1.15$ & NS \\
\hline Total duration of stimulation (days) & $10.51 \pm 1.9$ & $10.5 \pm 2.7$ & NS \\
\hline Total dose of gonadotrophins (IU) & $3724.22 \pm 498.05$ & $3422.97 \pm 391.46$ & NS \\
\hline
\end{tabular}

Table 2: Comparison of results of antagonist/agonist protocol.

\begin{tabular}{|llll|}
\hline & AP & LP & Statistical difference \\
\hline No. of oocytes retrieved & $3.11 \pm 0.71$ & $3.18 \pm 0.65$ & NS \\
\hline M II oocytes & $2.37 \pm 1.13$ & $2.33 \pm 0.72$ & NS \\
\hline Grade I embryos & $2.19 \pm 1.51$ & $2.17 \pm 1.1$ & NS \\
\hline No. of embryos transferred & $1.9 \pm 0.80$ & $1.8 \pm 0.6$ & NS \\
\hline Pregnancy rate per cycle & $37.7 \%$ & $32.6 \%$ & NS \\
\hline
\end{tabular}


Of the 99 patients considered with $\mathrm{AMH} \leq 1.5$ and $\mathrm{AFC}$ $\leq 4$ who underwent IVF between May 2019 and Mar 2020, 53 patients underwent the antagonist protocol and 46 underwent the GnRH agonist long protocol. Out of the 53 patients on antagonist protocol, 20 had a positive pregnancy test, and out of the 46 patients on agonist protocol, 15 were positive for pregnancy. Thus, implantation rates of $37.7 \%$ and $32.6 \%$ were found in antagonist and agonist protocols, respectively (Table 2).

The pregnancy rate was higher in the antagonist group, but the difference was not statistically significant. Embryo transfer was not done in two patients of the long agonist protocol and one patient of the antagonist protocol due to nil retrieval of oocytes. The age of these cases was between 39 and 42 years with $\mathrm{AMH}$ values ranging from $0.4 \mathrm{ng} / \mathrm{mL}$ to $0.6 \mathrm{ng} / \mathrm{mL}$.

\section{DISCUSSION}

An adequate stimulation protocol should assist the development and retrieval of an adequate number of mature oocytes. ${ }^{27}$ The number of retrieved oocytes in response to $\mathrm{COH}$ is a pivotal factor since it is an independent predictor of success of the cycle. Patients with POR are challenging to treat as the number of retrieved oocytes is very low in such cases, decreasing the overall chances of success and leading to the repeated cancellation of a cycle. ${ }^{28}$

AMH and AFC can individually be used as a standalone test for predicting POR. Presently, there is no consensus on the definition of POR and no cut-off value of a hormonal test or ultrasonographic finding to predict poor response. $^{29}$

In this study, we took an AMH value of $\leq 1.5 \mathrm{ng} / \mathrm{mL}$ and $\mathrm{AFC} \leq 4$ as POR and compared the efficacy of the long agonist and antagonist protocols in such cases.

Introduction of the GnRH antagonist in the field of assisted reproductive techniques is quite recent compared with the GnRH agonist, which has been in use in IVF cycles since the 1980s. ${ }^{30}$ The introduction of the GnRH antagonist was a new of hope for patients with POR. Due to its short inhibitory effect on pituitary, it was expected not to decrease the ovarian response during $\mathrm{COH}^{31}$ The $\mathrm{GnRH}$ antagonist does not inhibit early folliculogenesis due to late introduction in the cycle, which is an added advantage for patients with a limited cohort of follicles. ${ }^{32}$ The GnRH agonist, on the other hand, is administered for a longer duration throughout the cycle, competitively blocking the ovarian FSH receptors and suppressing the ovarian response to the gonadotropins. ${ }^{33}$ However, most of the studies have not found any difference in response to either protocols..$^{8,22,30,34}$ Several studies found that the total duration of stimulation and total dosage of gonadotropins in the antagonist group was much less than that in long agonist group. ${ }^{35-40}$ In this study, the duration of stimulation was same in both the groups. Although the total gonadotropins used was higher in the antagonist group, the difference was not statistically significant. The number of retrieved oocytes was higher in the agonist group in some studies, the antagonist group in some studies, and similar in others. , $8,22,37-40^{\text {The number of }}$ retrieved oocytes in our study were also similar in both groups. Higher pregnancy rates have been reported in antagonist group in some studies..$^{8,30,39}$ However, some research refutes this and claim to have found better result in the agonist group. . $2,40-42$ This study found higher pregnancy rates in the antagonist groups as compared with the agonist group; however, the difference was not statistically significant. Cycle cancellation was found to be less in the antagonist group and similar results were also observed in our study. ${ }^{39}$

\section{CONCLUSION}

Nowadays, the number of patients with POR has substantially increased in all IVF clinics due to late marriage and delayed childbearing. Despite extensive research, multiple stimulation protocols, and the addition of adjuncts, the treatment of patients with POR remains challenging to treat. Due to the reduced quantity and quality of oocytes in a patient with POR, it remains a major poor prognostic factor. POR, as predicted by different tests, indicates that pregnancy is less likely, but not impossible. The diagnosis and best protocol in patients with POR remain debatable. All protocols aim at achieving maximum good quality oocytes and embryos. Patients with POR need an aggressive approach and proper counselling and support. A lot of work is still needed to customise $\mathrm{COH}$ protocols for patients with POR depending on different cycle characteristics.

\section{Funding: No funding sources}

Conflict of interest: None declared

Ethical approval: The study was approved by the Institutional Ethics Committee

\section{REFERENCES}

1. Sunkara SK, Rittenberg V, Raine-Fenning $\mathrm{N}$, Bhattacharya S, Zamora J, Coomarasamy A. Association between the number of eggs and live birth in IVF treatment: an analysis of 400135 treatment cycles. Hum Reprod. 2011;26:1768-74.

2. De Geyter C, Fehr P, Moffat R, Gruber IM, von Wolff M. Twenty years' experience with the Swiss data registry for assisted reproductive medicine: outcomes, key trends and recommendations for improved practice. Swiss Med Wkly. 2015; 145:w14087.

3. Drakopoulos P, Blockeel C, Stoop D, Camus M, de Vos M, Tournaye $\mathrm{H}$, et al. Conventional ovarian stimulation and single embryo transfer for IVF/ICSI. How many oocytes do we need to maximize cumulative live birth rates after utilization of all fresh and frozen embryos? Hum Reprod. 2016;31:370-6. 
4. Esteves SC, Roque M, Bedoschi GM, Conforti A, Humaidan P, Alviggi C. Defining low prognosis patients undergoing assisted reproductive technology: POSEIDON Criteria - The why. Front Endocrinol (Lausanne). 2018;9:461.

5. Practice Committee of the American Society for Reproductive Medicine. Testing and interpreting measures of ovarian reserve: a committee opinion. Fertil Steril. 2015;103:e9-17.

6. Tal R, Seifer DB. Ovarian reserve testing: a user's guide. Am J Obstet Gynecol. 2017;217(2):105-234.

7. Rasool S, Shah D. Fertility with early reduction of ovarian reserve: the last straw that breaks the Camel's back. Fertil Res Pract. 2017;3:15.

8. Cheung LP, Lam PM, Lok IH, Tak-Yu Chiu T, Yeung SY, et al. GnRH antagonist versus long GnRH agonist protocol in poor responders undergoing IVF: a randomized controlled trial. Human Reprod. 2005;20(3):616-21.

9. Akman MA, Erden HF, Tosun SB, Bayazit N, Aksoy $\mathrm{E}$, Bahceci $\mathrm{M}$. Addition of GnRH antagonist in cycles of poor responders undergoing IVF. Hum Reprod. 2000;15:2145-7.

10. Patrizio P, Vaiarelli A, Setti L, Tobler KJ, Shoham $\mathrm{G}$, Leong M, et al. How to define, diagnose and treat poor responders? Responses from a worldwide survey of IVF clinics. Reprod Biomed Online. 2015;30:581-92.

11. Oudendijk JF, Yarde F, Eijkemans MJ, Broekmans FJ, Broer SL. The poor responder in IVF: is the prognosis always poor?: a systematic review. Hum Reprod Update. 2012;18:1-11.

12. Lazer T, Dar S, Shlush E, Al Kudmani BS, Quach K, Sojecki A, et al. Comparison of IVF outcomes between minimal stimulation and high-dose stimulation for patients with poor ovarian reserve. Int J Reprod Med. 2014:Article ID 581451.

13. Kyrou D, Kolibianakis EM, Venetis CA, Papanikolaou EG, Bontis J, Tarlatzis BC. How to improve the probability of pregnancy in poor responders undergoing in vitro fertilization: a systematic review and meta-analysis. Fertil Steril. 2009;91(3):749-66.

14. Vollenhoven B, Osianlis T, Catt J. Is there an ideal stimulation regimen for IVF for poor responders and does it change with age? J Assist Reprod Genet. 2008;25(11-12):523-9.

15. Verberg MF, Eijkemans MJ, Macklon NS, Heijnen EM, Baart EB, Hohmann FP, et al. The clinical significance of the retrieval of a low number of oocytes following mild ovarian stimulation for IVF: a meta-analysis. Hum Reprod Update. 2009;15:5-12.

16. Polyzos NP, Sunkara SK. Sub-optimal responders following controlled ovarian stimulation: an overlooked group? Hum Reprod. 2015;30:2005-8.

17. Sharara FI, Scott RT, Seifer DB. The detection of diminished ovarian reserve in infertile women. Am J Obstet Gynecol. 1998;179:804-12.

18. Gallos ID, Eapen A, Price MJ, Sunkara SK, Macklon NS, Bhattacharya S, Khalaf Y, Tobias A, Deeks JJ,
Rajkhowa M, Coomarasamy A. Controlled ovarian stimulation protocols for assisted reproduction: a network meta-analysis. Coch Data Syst Rev. 2017;2017(3):CD012586.

19. Yu R, Jin H, Huang X, Lin J, Wang P. Comparison of modified agonist, mild-stimulation and antagonist protocols for in vitro fertilization in patients with diminished ovarian reserve. J Inter Med Res. 2018;46(6):2327-37.

20. Lainas TG, Sfontouris IA, Venetis CA, Lainas GT, Zorzovilis IZ, Tarlatzis BC, et al. Live birth rates after modified natural cycle compared with highdose FSH stimulation using GnRH antagonists in poor responders. Human Reprod. 2015;30(10):232130 .

21. Sbracia M, Farina A, Poverini R, Morgia F, Schimberni M, Aragona C. Short versus long gonadotropin-releasing hormone analogue suppression protocols for superovulation in patients $\geq$ 40 years old undergoing intracytoplasmic sperm injection. Fertil Steril. 2005;84(3):644-8. .

22. Sibai H, Elgindy EA, Mostafa MI. ICSI cycles in women over 40 years: analysis of 3 different ovarian stimulation protocols. Middle East Fertil Society J. 2018;23(4):319-23.

23. Z. Pandian, A.R. McTavish, L. Aucott, M.P. Hamilton, S. Bhattacharya, Interventions for 'poor responders' to controlled ovarian hyper stimulation $(\mathrm{COH})$ in in-vitro fertilization (IVF). Cochrane Database Syst Rev. 2010;1:CD004379.

24. Haahr T, Esteves SC, Humaidan P. Poor definition of poor-ovarian response results in misleading clinical recommendations. Hum Reprod. 2018;33:979-80.

25. Haahr T, Esteves SC, Humaidan P. Individualized controlled ovarian stimulation in expected poorresponders: an update. Reprod Biol Endocrinol. 2018;16:20.

26. Orvieto R, Patrizio P. GnRH agonist versus GnRH antagonist in ovarian stimulation: an ongoing debate. Reprod Biomed Online. 2013;26(1):4-8.

27. Griesinger G, Diedrich K, Tarlatzis BC, Kolibianakis EM. GnRH-antagonists in ovarian stimulation for IVF in patients with poor response to gonadotrophins, polycystic ovary syndrome, and risk of ovarian hyperstimulation: a meta-analysis. Reprod Biomed Online. 2006;13(5):628-38.

28. Tal, R, Tal, O, Seifer BJ. Antimüllerian hormone as predictor of implantation and clinical pregnancy after assisted conception: a systematic review and metaanalysis. Fertil Steril. 2015;103:119-30.

29. Pastore LM, Christianson MS, Stelling J, Kearns WG, Segars JH. Reproductive ovarian testing and the alphabet soup of diagnoses: DOR, POI, POF, POR, and FOR. J Assisted Reprod Genetics. 2018;35(1):17-23.

30. Lai Q, Zhang H, Zhu G, Li Y, Jin L, He L, et al. Comparison of the GnRH agonist and antagonist protocol on the same patients in assisted reproduction during controlled ovarian stimulation 
cycles. Int $\mathrm{J}$ Clin Experimental Pathol. 2013;6(9):1903.

31. Mo Y, Peng P, Zhou R, He Z, Huang L, Yang D. Regulation of gonadotropin-releasing hormone $(\mathrm{GnRH})$ receptor-I expression in the pituitary and ovary by a GnRH agonist and antagonist. Reprod Sci. 2010;17(1):68-77.

32. Howles CM. The place of gonadotrophin-releasing hormone antagonists in reproductive medicine. Reproductive BioMedicine Online. 2002;4(3):64-71.

33. Lavorato HL, Oliveira JB, Petersen CG, Vagnini L, Mauri AL, Cavagna M, et al. GnRH agonist versus GnRH antagonist in IVF/ICSI cycles with recombinant LH supplementation: DNA fragmentation and apoptosis in granulosa cells. Eur $\mathbf{J}$ Obstet Gynecol Reprod Biol. 2012;165:61-5.

34. Bodri D, Vernaeve V, Guillen JJ, Vidal R, Figueras F, Coll O. Comparison between a GnRH antagonist and a GnRH agonist flare-up protocol in oocyte donors: a randomized clinical trial. Hum Reprod. 2006;21:2246-51.

35. $\mathrm{Pu} \mathrm{D,} \mathrm{Wu} \mathrm{J,} \mathrm{Liu} \mathrm{J.} \mathrm{Comparison} \mathrm{of} \mathrm{GnRH} \mathrm{antagonist}$ versus GnRH agonist protocol in poor ovarian responders undergoing IVF. Hum Reprod. 2011;26:2742-9.

36. Franco JG, Baruffi RL, Mauri AL, Petersen CG, Felipe V, Cornicelli J, et al. GnRH agonist versus GnRH antagonist in poor ovarian responders: a metaanalysis. Reprod Biomed Online. 2006;13(5):618-27.

37. Malmusi S, La Marca A, Giulini S, Xella S, Tagliasacchi D, Marsella T, et al. Comparison of a gonadotropin-releasing hormone $(\mathrm{GnRH})$ antagonist and GnRH agonist flare-up regimen in poor responders undergoing ovarian stimulation. Fertil Steril. 2005;84(2):402-6.
38. Xiao J, Chang S, Chen S. The effectiveness of gonadotropin-releasing hormone antagonist in poor ovarian responders undergoing in vitro fertilization: a systematic review and meta-analysis. Fertil Steril. 2013;100:1594-601.e1-9.

39. Roberto M, Donatella C, Vincenza D, Carla T, Antonio P, Massimo M. GnRH antagonist in IVF poor-responder patients: results of a randomized trial. Reprod Biomed Online 2005;11:189-93.

40. Orvieto R, Homburg R, Meltcer S, Rabinson J, Anteby EY, Scharf S. GnRH agonist versus GnRH antagonist in controlled ovarian hyperstimulation: their role in patients with an unfavorable prognosis a priori. Fertil Steril. 2009;91:1378-80.

41. Orvieto R, Rabinson J, Meltcer S, Homburg R, Anteby E, Zohav E. GnRH agonist versus GnRH antagonist in ovarian stimulation: is the emperor naked?. Clin Exp Obstet Gynecol. 2006;33:197-9.

42. Prapas Y, Petousis S, Dagklis T, Panagiotidis Y, Papatheodorou A, Assunta I, et al. GnRH antagonist versus long GnRH agonist protocol in poor IVF responders: a randomized clinical trial. Eur J Obstet Gynecol Reprod Biol. 2013;166(1):43-6.

Cite this article as: Rai $\mathrm{S}$, Khandavalli Y, Lodha $\mathrm{RS}$. Is there a perfect protocol for patients with low ovarian reserve: a retrospective study comparing antagonist or agonist protocol in patients with low ovarian reserve. Int J Reprod Contracept Obstet Gynecol 2020;9:3151-6. 